\title{
SUSCEPTIBILIDAD A LA EROSIÓN EN DOS AGROECOSISTEMAS ALTOANDINOS DEL CAUCA
}

\author{
EROSION SUSCEPTIBILITY IN TWO ANDEAN CAUCA AGROECOSYSTEMS
}

\author{
${ }^{1}$ Fernando Andrés Muñoz Gómez; ${ }^{2}$ Apolinar Figueroa Casas; ${ }^{3}$ Edier Humberto Pérez; ${ }^{4}$ Edwin Rengifo Cañizalez.
}

Fecha de recibido: Febrero 3 de 2010

Fecha de aceptado: Junio 9 de 2010

Correspondencia: Grupo de Estudios Ambientales, Universidad del Cauca, Popayán (Cauca),

correo electrónico:femunoz@unicauca.edu.co

\section{RESUMEN}

Las características ambientales de los suelos en los agroecosistemas altoandinos los hacen susceptibles a procesos erosivos generados por la intensidad y duración de las lluvias. Estos efectos se intensifican al disminuir la cobertura vegetal en la etapa de preparación del suelo para el cultivo. Por estas razones se trabajo sobres cultivos implantados y se determinó la cantidad de suelo desplazado por escorrentía superficial en lluvia simulada a $100 \mathrm{~mm} \mathrm{~h}-1$ de intensidad, obteniéndose una media estadística de suelo erodado en terreno para cultivo de $2.6 \mathrm{~g}$ y en terreno con pastura de $0.2 \mathrm{~g}$. Esta información permitió desarrollar una evaluación ambiental, usando la matriz de FEARO (oficina federal de revisión y análisis ambientales), con la cual se identificó que los componentes ambientales más afectados son el suelo, flora y la fauna, generando un cambio en las características propias de este ecosistema.

Palabras claves: Erodabilidad, escorrentía, Agro ecosistema, suelos ándicos.

\section{ABSTRACT}

The environmental characteristics of the soils in the Andean agro ecosystems make them susceptible to erosion caused by the intensity and duration of the rains. These effects are intensified by reducing vegetation cover in the stage of soil preparation for cultivation. For these reasons we worked on crops implanted and also it was determined the amount of soil displaced by surface runoff in simulated rainfall at $100 \mathrm{~mm} \mathrm{h-1}$ of intensity, yielding a statistical average of eroded soil on the ground for cultivation of $2.6 \mathrm{~g}$ and pasture land with $0.2 \mathrm{~g}$. This information allowed the development of an environmental assessment, using the matrix of FEARO (Federal Office for review and environmental analysis), with which it was identified soil, flora and fauna as the most affected environmental components, causing a change in the characteristics of this ecosystem.

Key words: Erodability, run-off, infiltration, Agro ecosystem, Andean soil.

\section{INTRODUCCIÓN}

Los suelos ándicos presentan gran cantidad de materiales orgánicos especialmente aquellos presentes en las zonas de vida muy húmeda y con alta influencia de cenizas volcánicas. Los cambios en las propiedades texturales relacionadas con la cohesión del material, la permeabilidad en estudios realizados por el Ministerio de Agricultura de los Estados Unidos (USDA) en (Diez, s.f) comprueban que los suelos erosionables corresponden a las texturas intermedias (fracción de limo abundante) del tipo fr (franco), frLi (franco limoso). De igual forma los altos niveles de precipitación, el tipo de pendiente, su exposición y las actividades agropecuarias desarrolladas; generan altos volúmenes de escorrentía superficial entre otros procesos erosivos.

\footnotetext{
${ }^{1}$ Grupo de Estudios Ambientales, Universidad del Cauca, Popayán, Cauca, Colombia.

${ }^{2}$ Grupo de estudios ambientales, Universidad del Cauca (GEA)

${ }^{3}$ facultad de educación; Universidad del cauca

${ }^{4}$ Grupo de Estudios Ambientales, Universidad del Cauca, Popayán
} 
La degradación ambiental continúa siendo uno de los problemas más comunes en áreas rurales causadas principalmente por actividades humanas ligadas a sistemas de producción extensiva e intensiva, bien sea de tipo agrícola, ganadero o minero. La disminución de coberturas vegetales antes y después del cultivo, el aumento en la frecuencia de quemas y el sobre pastoreo, son algunos de los factores que conducen a la degradación de sus condiciones originales. Es el caso de cultivos limpios de papa y ganadería que dejan expuesto los suelos a procesos erosivos modificando severamente sus condiciones físicas, químicas, biológicas y la productividad de los ecosistemas por un continuo transporte de partículas y nutrientes necesarios para el desarrollo de las diferentes formas de vida. Debido principalmente a la baja capacidad de infiltración del suelo y la falta de cobertura vegetal que genera láminas de escorrentía arrastrando el suelo desprendido por las gotas de lluvia, como indican los resultados de (Morgan, 1986), una fuerte relación entre la escorrentía y el suelo erodado. La magnitud de este efecto se puede deducir de acuerdo a las investigaciones de Bennett 1939 en (Hudson, 1982) donde indica que bajo condiciones no alteradas son necesarias del orden de 300 años para producir $25 \mathrm{~mm}$ de suelo. Estas cifras también pueden ser comparadas con valores promedios de procesos de formación del suelo reportados por la (FAO, 1996) cercanas a una ton/ha/año.

De acuerdo al IDEAM, (1998) se están afectando cantidad y calidad de los recursos hídricos, porque la deforestación, las actividades agrícolas y domésticas reducen la regulación de los caudales, aceleran la erosión y generan contaminación de las aguas. En la actualidad Colombia cuenta con pocos estudios técnicos que permitan determinar y generalizar la cantidad de suelo que es desplazado por erosión hídrica en zonas altoandinas; y en especial el impacto generado sobre los agroecosistemas. Reportes del IDEAM, (2001) muestran que alrededor de un $48 \%$ del territorio nacional está impactado con algún grado de erosión y de este porcentaje un 14.2 \% está afectado con un grado de erosión muy alto. Para el Departamento del Cauca en la zona norte, (Suárez, 1982) concluye que el proceso erosivo ha llevado a la pérdida casi completa de la capa de cenizas volcánicas que antes cubrieron el área. En el Municipio de Totoró y en especial el corregimiento de Gabriel López se presentan procesos de erosión ligera, evidenciados por el fenómeno de reptación (pata de vaca), sobre pastoreo y cultivos limpios con la utilización de agentes químicos, maquinaria pesada, que impiden el crecimiento de vegetación asociada y unidos a otros agentes están incrementando la pérdida de suelo por escorrentía superficial. (E.O.T, 2002). Razón importante para evaluar y analizar de forma cuantitativa la cantidad de suelo erodado por escorrentía superficial, ante lluvias de alta intensidad en suelos ándicos sin cobertura y con cobertura de pastos identificando y evaluando los factores influyentes. Ya que en la zona de estudio la actividad más representativa de uso del suelo es el cultivo de papa, generando serios impactos sobre el ecosistema, debido a la preparación de tierras para cultivo, dejando expuesto el suelo a efectos de la lluvia, creando un gran movimiento de suelo y partículas con nutrientes como fosfatos, nitratos o cloruros por escorrentía hacia los cuerpos de agua.

\section{MATERIALES Y MÉTODOS}

El trabajo se desarrolló en la cordillera central Colombiana correspondiente a la región Andina en el Departamento del Cauca, Municipio de Totoró, Corregimiento de Gabriel López. Sus coordenadas geográficas son: 76o 18' 53" W; 20 $28^{\prime} 20^{\prime \prime} \mathrm{N}$. En la evaluación de la susceptibilidad a la erosión se utilizó un minisimulador de lluvias fundamentado en el sistema de Vaso de Mariotte diseñado por (Cobo, 1998), el cual permite tomar mediciones de: infiltración, suelo erodado y escorrentía superficial por efecto de las gotas de Iluvia. El área de evaluación en campo es $33 \mathrm{~cm}$ por $42 \mathrm{~cm}$ y una altura de emisión de gota de aproximadamente 1 metro. Complementado con el análisis en laboratorio de algunas propiedades físicas y químicas en suelos disturbados y sin disturbar; teniendo como precedente la metodología utilizada por (Torres, 2000), (Campo, 2004) y (Bravo, 2003) para el análisis de suelos. Se estableció un diseño experimental completamente al azar con tres repeticiones en cada una de seis parcelas, con rangos de pendiente $15 ; 25$ y $50 \%$, considerando el uso y manejo del suelo (pastura y suelo preparado para cultivo de papa), durante una temporalidad de 6 meses. Para realizar las simulaciones se estableció una intensidad de lluvia (100 mm h-1) de acuerdo con los datos suministrados por Instituto de Hidrología, Meteorología y Estudios Ambientales IDEAM en el Plan de Ordenamiento Territorial (POT, 2000), en una temporalidad de diez años; y un diámetro de gota de lluvia de $(2.75 \mathrm{~mm})$ más el tiempo requerido para cada simulación (30 minutos), con toma de muestra cada cinco minutos, para establecer un total de 108 muestreos de escorrentía, suelo erodado e infiltración. Se realizó una evaluación preliminar del efecto que tienen estas actividades sobre los diferentes componentes de un ecosistema altoandino, aplicando Pruebas de Mann-Whitney para uso de sueloy Duncan para las variables uso y tiempo, buscando diferencias entre tratamientos; además se utilizó el método de Matrices de FEARO propuesto por Figueroa, (1998) en el que asigna un color al efecto que ha causado una actividad de acuerdo a los conocimientos e información que tiene el investigador de forma cualitativa. 


\section{RESULTADOS Y DISCUSIÓN}

3.1. Propiedades físicas, químicas y su influencia en la erodabilidad del suelo.

En la tabla 1 se reportan los resultados de estas propiedades

Tabla 1. Propiedades físicas y químicas del suelo (Prueba U de Mann-Whitney)

\begin{tabular}{lrrr}
\hline \multicolumn{1}{c}{ Variables } & Pastura & Cultivo & \multicolumn{1}{c}{ Sig } \\
\hline \%Arena & 7,45 & 19,94 & 0,05 \\
\%Limo & 87,4 & 72,5 & 0,05 \\
\%Arcilla & 5,14 & 7,53 & 0,15 \\
Textura & $\mathrm{Li}$ & $\mathrm{FrLi}$ & \\
Porosidad total \% & 78,15 & 75,75 & 0,28 \\
Densidad Real g/cc & 2,19 & 2,17 & 0,12 \\
Densidad Aparente g/cc & 0,48 & 0,53 & 0,3 \\
Permeabilidad cm/seg & $4,5 \times 10-4$ & $3,3 \times 10-4$ & 0,44 \\
Humedad \% de campo & 152,8 & 95,8 & 0,44 \\
Materia Orgánica \% & 29,43 & 28,16 & 0,21 \\
pH & 4,92 & 4,96 & 0,1 \\
Al+3 & & & \\
Intercambiable(meq/100g) & 3,25 & 1,88 & 0,04 \\
\hline
\end{tabular}

Los valores de densidad real (Dr) y densidad aparente (Da) están dentro de un rango bajo para los dos sistemas. Se observa un leve incremento en Dr de Pastura, y Da de suelo desnudo, atribuido principalmente a las actividades agrícolas desarrolladas que destruyen la estructura terrosa del suelo y a la escasa cobertura vegetal, sin embargo, este incremento no es significativo. Los bajos valores de Da en ambos suelos explican el mayor espacio aéreo atribuible a su alta porosidad total, reflejando la facilidad de circulación de agua y aire; pues a medida que aumenta la densidad aparente se aumenta la compactación, se reducen la porosidad total, la humedad del suelo, la conductividad del agua a través del perfil y la actividad del los microorganismos.

La permeabilidad es baja en ambos sistemas, aún cuando no se observa diferencia significativa $($ sig=0.44) en el suelo de pasturas es ligeramente superior, manifestando que tiene un nivel ligeramente mayor de permeabilidad lo cual facilita la infiltración del agua, mientras que el suelo desnudo se puede saturar más fácilmente con lluvias de alta intensidad.

Los porcentajes de humedad de campo son altos debido a su alto contenido de $\mathrm{MO}$, la diferencia aún cuando no es significativa se explica posiblemente en la exposición que presenta el suelo desnudo ante el sol facilitando la evaporación y por ende menores valores de humedad que generan erosión eólica o hídrica, caso contrario al suelo con pastos donde la humedad se mantiene.
En ambos suelos predominan partículas de limo, correspondientes a minerales primarios los cuales no son fuente de nutrientes para el suelo, deben meteorizarse para tal fin. Se observa deficiencia de arcillas, minerales secundarios muy importantes porque son los más reactivos uniéndose a la $\mathrm{MO}$ del suelo para formar una verdadera bioestructura y generar estabilidad evitando erosión y escorrentía. En el suelo de pastura el contenido de limos es significativamente mayor generando así una textura limosa ocasionando mayor problema. El suelo de papa pertenece a la categoría de los francos apropiados para labores agrícolas, pero su deficiencia de arcillas agravado por el alto contenido de MO genera serios problemas de efecto Priming y erosión causada por el agua de escorrentía ricas en lodo, pudiendo modificarse la textura en su capa superficial; arrastre debido a que el alto contenido de $\mathrm{MO}$ es indicio de deficiencia de un adecuado proceso de humificación, predominando así la materia orgánica fresca fácilmente erosionable.

Los porcentajes de humedad de campo son altos debido a su alto contenido de $\mathrm{MO}$, la diferencia aún cuando no es significativa se explica posiblemente en la exposición que presenta el suelo desnudo ante el sol facilitando la evaporación y por ende menores valores de humedad que generan erosión eólica o hídrica, caso contrario al suelo con pastos donde la humedad se mantiene.

Por lo tanto podemos asumir que los suelos con predominios de arenas y limos presentan una baja estabilidad al impacto de las gotas de lluvia, por la baja cohesión de las partículas dentro de las unidades estructurales. Reflejando en la formación de sellos superficiales que generan un descenso en la permeabilidad del suelo. El contenido de materia orgánica a la profundidad de $0-5 \mathrm{~cm}$. es considerablemente alto y se presenta una leve diferencia no significativa entre los tratamientos que indica como las actividades agrícolas en suelo desnudo disminuyen el contenido de Materia Orgánica (MO) en función del tiempo e intensidad de uso del suelo al igual que la erosión en sus diferentes formas. El pH en los dos suelos indica una muy fuerte acidez atribuible no solamente al material parental, por la generación de niveles medio y altos de Al intercambiable, si no al alto contenido de $\mathrm{MO}$ como se evidencia de este análisis. Siendo el contenido de Al significativamente superior en el suelo de pastura donde el valor de $\mathrm{pH}$ es más bajo y el contenido de MO más alto, así sus diferencias con los correspondientes valores del suelo cultivado no sean significativas.

\section{Agroecosistema con suelo desnudo}

En suelo desnudo a comparación del agroecosistema de pastizal se generan mayores cantidades de escorrentía y 


\section{- 12 - Agroecosistemas altoandinos del Cauca}

suelo erodado, pero disminuye el volumen de infiltración (Tabla 2), ya que la exposición directa del suelo a la lluvia crea un sellamiento o saturación de los poros por impacto de las gotas de lluvia, causando un incremento de los flujos superficiales que desplazan los agregados o partículas del suelo.

Tabla 2. Medias marginales estimadas para suelo desnudo y pastura

\begin{tabular}{|c|c|c|c|c|c|}
\hline \multirow[b]{2}{*}{ Variable dependiente } & \multirow[b]{2}{*}{ Uso del Suelo } & \multirow[b]{2}{*}{ Media } & \multirow[b]{2}{*}{ Error típ. } & \multicolumn{2}{|c|}{ Intervalo de confianza al 95\%. } \\
\hline & & & & Límite inferior & $\begin{array}{l}\text { Límite } \\
\text { superior }\end{array}$ \\
\hline \multirow{2}{*}{ Escorrentía (mL) } & Cultivo & $902.426(\mathrm{a})$ & 71.221 & 761.034 & 1043,818 \\
\hline & Pastura & $603.611(a)$ & 71.221 & 462.219 & 745.003 \\
\hline \multirow{2}{*}{ Suelo Perdido (g) } & Cultivo & $2.566(\mathrm{a})$ & .212 & 2.145 & 2.986 \\
\hline & Pastura & $.225(\mathrm{a})$ & .212 & -.196 & .646 \\
\hline \multirow{2}{*}{ Infiltración (mL) } & Cultivo & $5080.537(a)$ & 101.477 & 4879.080 & 5281,994 \\
\hline & Pastura & $5626.296(\mathrm{a})$ & 101.477 & 5424.840 & 5827,753 \\
\hline
\end{tabular}

\section{Agroecosistema de Pastura}

La cobertura vegetal sea viva o muerta en el agroecosistema pastura (Tabla 2) con dominancia de las familias Asteraceae, Clussiaceae, Fabaceae, Poaceae, Poligomnaceae y Rosaceae, son el elemento natural de protección del suelo frente a la fuerza erosiva de las precipitaciones y la escorrentía. Ya que la vegetación disminuye el golpe directo de las gotas de lluvia en su follaje y el sistema radicular facilita la infiltración de agua, sirviendo como un medio de amarre para el suelo y al mismo tiempo la descomposición tanto de tallos, hojas y raíces generan una película protectora del suelo ante los agentes erosivos y ayuda en la formación del mismo.

\section{Descripción de la variable escorrentía y suelo erodado} respecto al tiempo de evaluación.

Mediante la prueba de Duncan (Tabla 3) se analizaron las variables Escorrentía, Suelo erodado e Infiltración en los seis meses de estudio generando subconjuntos con las medias que están relacionadas. Se observa que los valores más bajos de escorrentía corresponden a los suelos de cultivo para los meses de Enero, Marzo y Abril y al de pastura para los meses de Enero, Febrero, Abril, Mayo y Junio. Los valores más altos corresponden al suelo de cultivo en los meses de Mayo y Junio. Lo cual está muy relacionado con los meses que presentaron mayor precipitación natural creando un incremento en la humedad del suelo o saturación del mismo.

Rev. Invest. Univ. Quindío (20): 9- 17. Armenia - Colombia
Tabla 3. Prueba de Duncan para Escorrentía.

\begin{tabular}{lrcccr}
\hline & \multicolumn{5}{c}{ Subconjunto para alfa $=.05$} \\
INTER ACC & N & 1 & 2 & 3 & 4 \\
\hline Cult.Marzo & 9 & 213,89 & & & \\
Cult.Abril & 9 & 213,89 & & & \\
Past.Febrero & 9 & 349,44 & 349,44 & & \\
Past.Enero & 9 & 407,78 & 407,78 & 407,78 & \\
Past.Junio & 9 & 558,89 & 558,89 & 558,89 & \\
Cult.Enero & 9 & 692,78 & 692,78 & 692,78 & \\
Past.Mayo & 9 & 731,11 & 731,11 & 731,11 & \\
Past.Abril & 9 & 731,11 & 731,11 & 731,11 & \\
Past.Mayo & 9 & & 843,33 & 843,33 & \\
Cult.Febrero & 9 & & & 957,89 & \\
Cult.Junio & 9 & & & & 1634,44 \\
Cult.Mayo & 9 & & & & 1701,67 \\
Sig. & & .074 & .085 & .054 & .785 \\
\hline
\end{tabular}

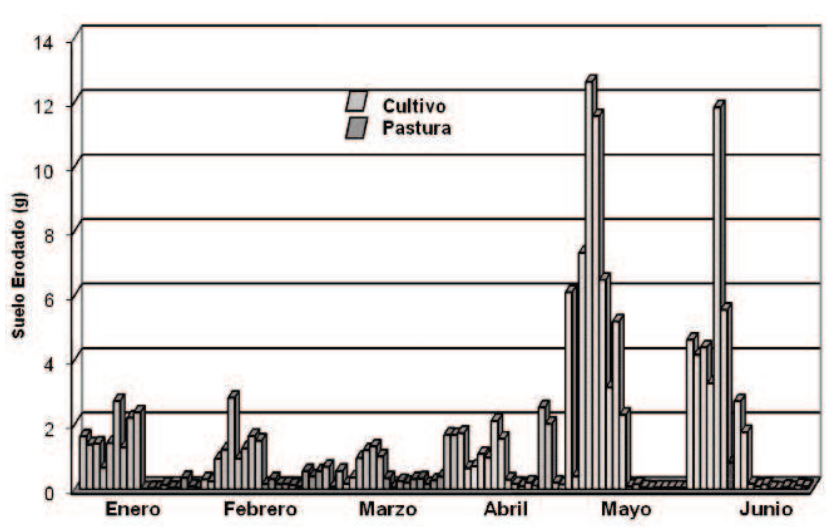

Figura 1. Suelo erodado para una intensidad de lluvia de $100 \mathrm{~mm} / \mathrm{h}$ por mes 
La figura 1 muestra el comportamiento que tuvo el suelo erodado en los seis meses de muestreo para los dos suelos; donde se puede observar valores hasta de $12 \mathrm{~g}$ de suelo erodado en un area de $40 \times 32 \mathrm{~cm}$ para el mese de mayo, que coinciden con los meses de mayor precipitacion para la zona, caso contrario del sistema de pastura que no sobrepasan los $2.1 \mathrm{~g}$. es aquí donde se puede evidenciar la funcion que cumple la cobertura vegetal en este tipo de agroecosistemas pues el suelo al estar saturado de agua genera altos niveles de escorrenti y suelo erodado.

La prueba de Duncan para suelo erodado (Tabla 4) en el tiempo de muestreo genera tres subconjuntos bien definidos. Los menores rangos de suelo desplazado se observan especialmente para el sistema de pastura en todos los meses de muestreo, lo mismo que para suelo desnudo en los meses de enero, marzo y agosto. El conjunto 2 presento $4.34 \mathrm{~g}$ de suelo erodado en suelo desnudo para el mese de junio, significativamente superior al anterior grupo. El valor más alto lo presenta el suelo desnudo con $6.12 \mathrm{~g}$ en el mes de mayo, existiendo una relación directa entre suelo erodado y escorrentía.

Tabla 4. Prueba de Duncan para Suelo erodado.

\begin{tabular}{lcccc}
\hline & \multicolumn{4}{c}{ Subconjunto para alfa $=.05$} \\
INTER ACC & $\mathrm{N}$ & 1 & 2 & 3 \\
\hline Past.Mayo & 9 &, 05 & & \\
Past.Junio & 9 &, 07 & & \\
Past.Enero & 9 &, 08 & & \\
Past.Marzo & 9 &, 25 & & \\
Past.F ebrero & 9 &, 27 & & \\
Past.Agosto & 9 &, 63 & & \\
Cult.Marzo & 9 &, 70 & & \\
Cult.Enero & 9 & 1,20 & & \\
Cult.Agosto & 9 & 1,35 & & \\
Cult.Enero & 9 & 1,67 & & \\
Cult.Junio & 9 & & 4,34 \\
Cult.Mayo & 9 & & & 1,00 \\
Sig. & &, 06 & & \\
\hline
\end{tabular}

Basado en las unidades de medida utilizadas internacionalmente para este factor como son toneladas/hectárea/mes se logró extrapolar la cantidad de suelo erodado que se generaría por una intensidad de lluvia de $100 \mathrm{~mm}$ h-1 durante los meses objeto de estudio (Figura 2). Se puede demostrar que en suelo desnudo la cantidad de suelo erodado para seis meses (1.2 ton/ha/mes) sobrepasa la tasa de formación en suelos ándicos. (Nearing. etal. 2005) Postula que el aumento de las precipitaciones asociadas con la intensidad es muy importante en relación con los posibles cambios en las tasas de erosion y el cambio climatico.

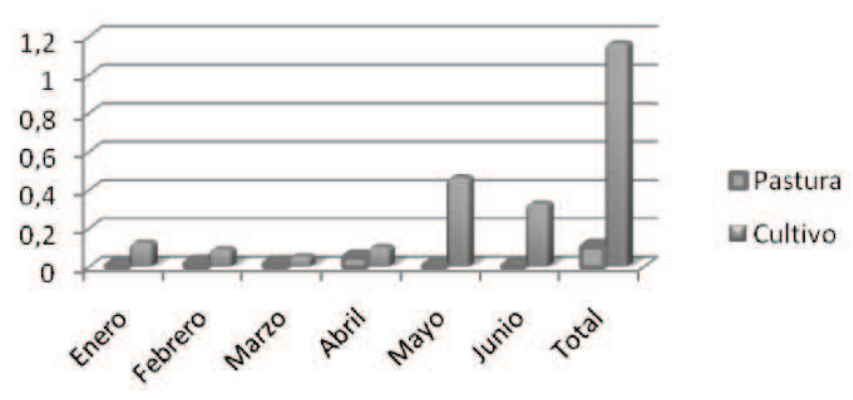

Figura 2. Extrapolación de suelo erodado en ton/ha/mes

\section{Descripción de la variable Infiltración}

La tabla 5 agrupa los valores en cuatro subconjuntos. En el subconjunto 1 están los volúmenes más bajos de infiltración para cultivo en los meses de mayo y junio. Los subconjuntos 2,3 y 4 están muy relacionados entre si, y presentan los volúmenes mas altos para el sistema de suelo desnudo en el mes de mayo. La baja infiltración en mayo y junio para el sistema de cultivo, se debe principalmente a que se ha superado la capacidad de absorción del suelo, creando un sistema húmedo y saturado que obstaculiza el paso del agua. La diferencia en el comportamiento de la infiltración en los tratamientos de suelo desnudo y pastura es creada principalmente por las prácticas agrícolas que del suelo se hacen, las cuales disminuyen a largo plazo los macroporos y por ende la infiltración.

Tabla 5. Prueba de Duncan para Infiltración

\begin{tabular}{lrrrrr}
\hline & \multicolumn{5}{c}{ Subconjunto para alfa $=.05$} \\
INTERACC & $\mathrm{N}$ & 1 & 2 & 3 & \multicolumn{1}{c}{4} \\
\hline Cult.Mayo & 9 & 3830,56 & & & \\
Cult.Junio & 9 & 4282,78 & & & \\
Cult.Febrero & 9 & & 5015,44 & & \\
Past. Mayo & 9 & & 5033,33 & & \\
Cult.Enero & 9 & & 5378,33 & 5378,33 & \\
Past.Mayo & 9 & & 5448,33 & 5448,33 & \\
Past. Junio & 9 & & 5661,11 & 5661,11 & 5661,11 \\
Cult.Abril & 9 & & 5667,78 & 5667,78 & 5667,78 \\
Past.Abril & 9 & & 5707,22 & 5707,22 & 5707,22 \\
Past. Febrero & 9 & & & 5941,67 & 5941,67 \\
Past.Enero & 9 & & & 5966,11 & 5966,11 \\
Cult.Mayo & 9 & & & & 6308,33 \\
Sig. & & .200 & .091 & .153 & .110 \\
\hline
\end{tabular}

Efecto de la pendiente sobre escorrentía, suelo erodado e infiltración

Con el análisis estadístico se determino que las pendientes del terreno no influyen en la escorrentía, el suelo erodado e infiltración (significancia $\mathrm{p}>0.05$ ); por lo cual se puede asumir que no hay efecto o su comportamiento es similar. Este fenómeno puede explicarse porque las dimensiones en las bandejas de escorrentía y los flujos superficiales, no 
alcanzan a tener altas velocidades; ya que las pendientes ejercen su mayor efecto con respecto a la erosión de los suelos cuando su longitud aumenta creando mayores niveles de escorrentía y las partículas adquieren mayor energía cinética a medida que avanzan a lo largo del terreno.

\section{Evaluación ambiental de la ganadería extensiva (Figura 3 )}

Según la matriz (Figura 3) el aire se ve afectado adversamente por las quemas, talas y adecuaciones del terreno, por la producción de gran cantidad de $\mathrm{CO} 2$ que es arrojado a la atmósfera; de igual forma la generación de ruido con la utilización de motosierras, hachas y maquinaria pesada. Impactando directamente la fauna, causando un desplazamiento de las especies presentes, pérdida de las comunidades vegetales, en los estratos arbóreos y arbustivos por un incremento en la velocidad del viento.

El agua presenta un efecto adverso porque produce el cambio de cobertura vegetal boscosa a pastizales, cuando se utiliza tala, quema y pastoreo del ganado que genera compactación del suelo, reduciendo la capacidad de infiltración y aumentando los niveles de escorrentía superficial. Creando cambios severos en la regulación hídrica, en propiedades físicas, químicas y biológicas por sedimentación, que alteran las zonas riparianas y las dinámicas tróficas (pérdida de habitas y nichos) de las comunidades acuáticas, especialmente en la región andina donde se presentan rangos elevados de precipitación.

El suelo es el componente del ecosistema con mayor impacto, siendo significativamente adverso reflejado en procesos de compactación o efecto de pata de vaca que han producido la pérdida casi total del horizonte Aoo, cambios en la macro porosidad, permeabilidad y estabilidad estructural; incrementando la escorrentía superficial, suelo erodado y posibles remociones mázales. (Francis 2009) propone que la erosión limita severamente las cosechas y reduce las entradas de carbono al suelo.

La flora y fauna presentan un efecto significativo adverso para este ecosistema, por que casi la totalidad de la vegetación ha sido reemplazada por cultivos semestrales y luego por pastos permanentes, generando fragmentación de ecosistemas, perdida de hábitats, nichos y diversidad de especies; alterando los procesos sucesionales naturales. Creando un ambiente propicio para la aparición de especies oportunistas. La falta de cobertura vegetal nativa y el libre acceso de los animales a los cursos de agua, generan mayor sedimentación (sólidos disueltos en el agua) y el aporte de excretas incrementan en gran medida los coliformes fecales, afectando la calidad del recurso hídrico.

Esta actividad tiene un efecto significativamente benéfico en la economía de la población, pues es fuente de ingresos con la comercialización de lácteos y todos los procesos que se derivan a partir de ella, pues están encaminados a mejorar la producción. Pero tiene un impacto adverso sobre el paisaje y el ecosistema al expandir la frontera pecuaria, con el propósito de tener más campos para pastoreo apropiándose de los recursos naturales en áreas donde su vocación es totalmente forestal y de conservación, con la intención de tener mayores ingresos en detrimento de la calidad de estos ecosistemas.

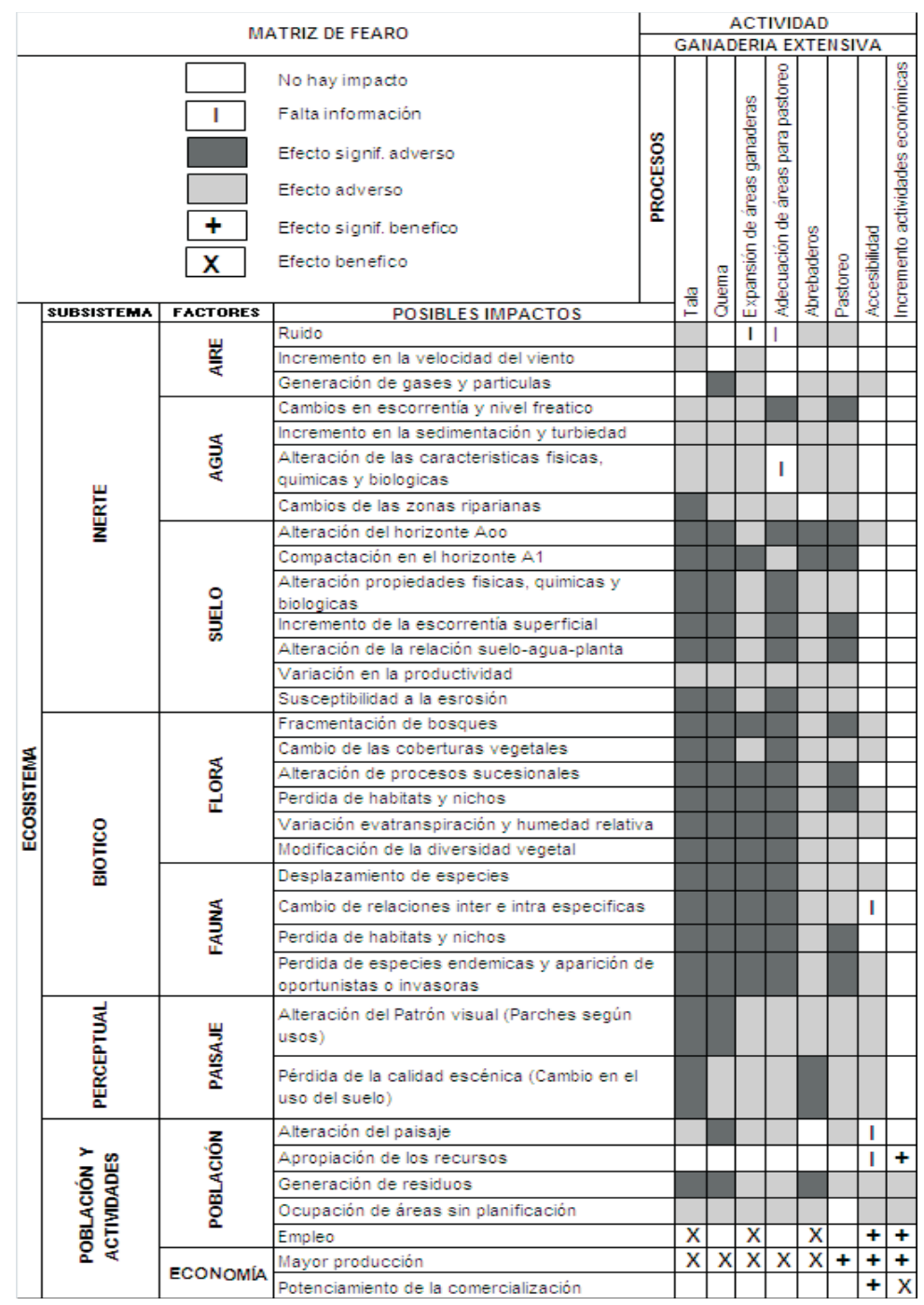

Figura 3. Matriz de FEARO para ganadería extensiva Suelo desnudo para el cultivo de papa (figura 4) 
El componente aire tiene un impacto adverso por las quemas y la gran cantidad de nutrientes que se volatilizan, alterando las relaciones de los ciclos biogeoquímicos y las características microclimáticas del sector, de igual forma la generación de ruido y gases por la maquinaria y otras actividades en la preparación de tierras produce un alejamiento del la fauna presente. Así mismo la eliminación de la cobertura vegetal incrementa la velocidad del viento.

Los altos niveles de suelo erodado tienen un efecto adverso sobre el agua pues las practicas agrícolas en el cultivo de papa, generan sedimentación de las fuentes de agua; como lo es la Cienaga de Calvachey, el rio Palacé y las zonas riparianas de estos sistemas hídricos presentando colmatación ya que son los primeros depósitos de todo el material transportado.

La utilización del suelo desnudo para cultivos afecta este componente de forma significativamente adversa, pues para la implantación se elimina la totalidad de la cobertura vegetal, para luego hacer uso de maquinaria pesada o implementos de labranza en el volteo y disgregación del suelo. Generando la pérdida del horizonte Aoo; compactación casi irreversible en los estratos mas bajos; reducción del tamaño de los poros e interrupción de la conexión entre ellos, aumento de la temperatura del suelo, disminución de la humedad, pérdida de nutrientes y materia orgánica por insolación, viento y lluvia; alteración en los niveles de escorrentía, cambiando las características físicas, químicas y biológicas de suelos, repercutiendo en la relación suelo-aguaplanta y en la productividad de los mismo. (Díaz. 2008) indica que cuando el suelo tiene mayor profundidad promedio sus propiedades son mejores para el cultivo, pero también son mayores la erodabilidad y en consecuencia los riesgos de erosin potencial y actual.

El efecto más grande siendo significativamente adverso se encuentra en la flora, ya que es eliminada totalmente, llevando a la pérdida potencial de hábitats y nichos, sumado al proceso de fragmentación de ecosistemas altoandinos que modifican la diversidad vegetal e interrumpen las cadenas tróficas y el proceso sucesional propio de estos sistemas e incluso afecta las especies endémicas, causando cambios en los niveles de evapotranspiración y precipitación variando las condiciones ambientales propias de ecosistemas altoandinos.

Según información reportada por la población el efecto de esta actividad ha sido adverso ya que la expansión de la frontera tanto agrícola como pecuaria ha ido en detrimento de la calidad de vida de las personas por el cambio de coberturas vegetales, generación de residuos, apropiación de recursos sin tener en cuenta la aptitud que tienen los suelos y la alta sedimentación; se han deteriorado las fuentes de agua, suelos y las condiciones ambientales propias de estos ecosistemas haciéndose necesario la utilización de gran variedad de agroquímicos para suplir las deficiencias del suelo y combatir la plagas. Para la actividad económica el efecto es benéfico en general pues todos los procesos realizados conducen a una mayor producción y un potenciamiento de la actividad comercial por la explotación de los recursos, logrando situar esta zona como una de las más productivas en el Departamento del Cauca en cuanto a papa y lácteos se refiere, generando una oportunidad laboral para los habitantes.

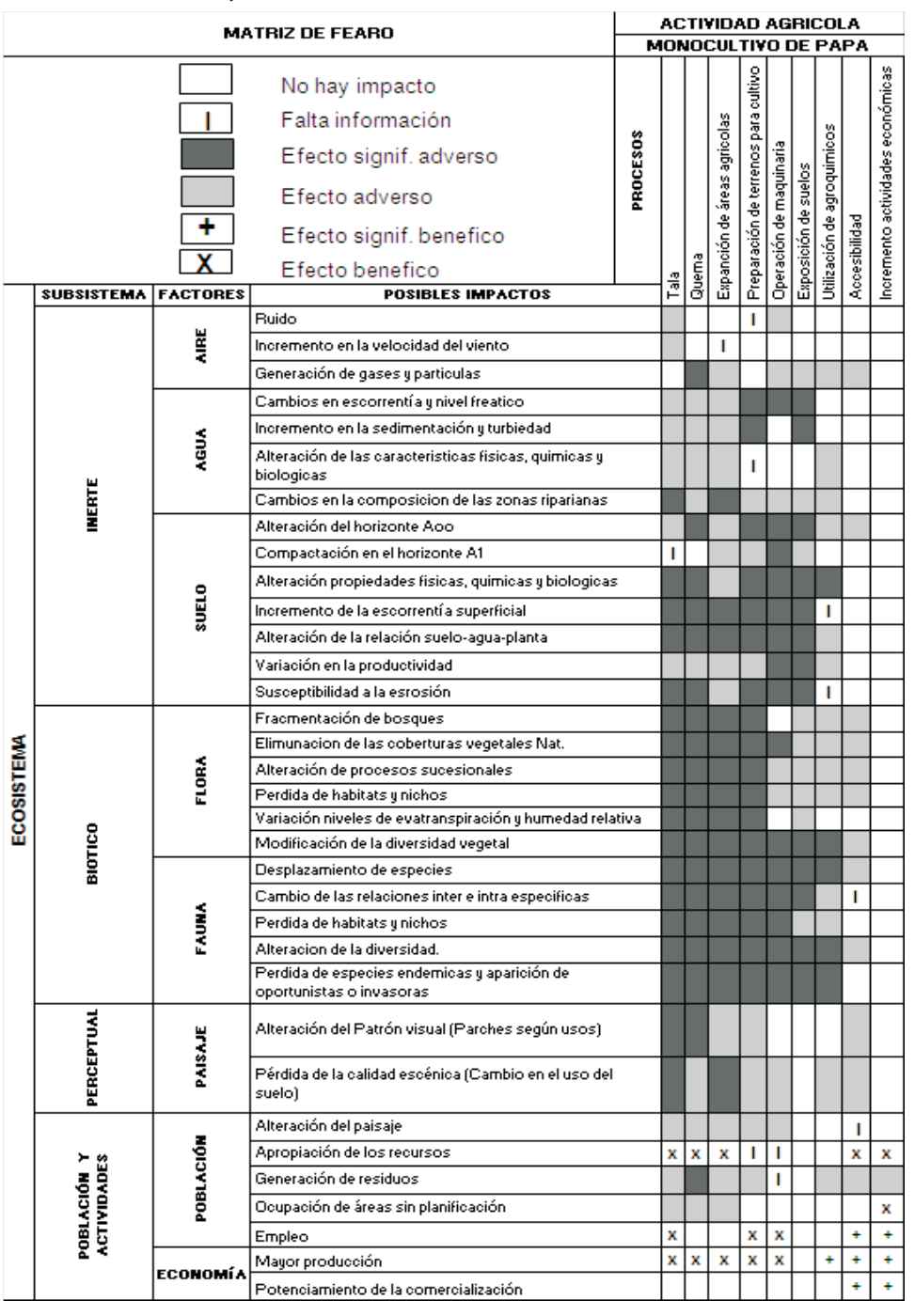

Figura 4. Matriz de FEARO para el monocultivo de papa 


\section{- 16 - Agroecosistemas altoandinos del Cauca}

\section{CONCLUSIONES}

Los bajos niveles de escorrentía en el sistema de cultivo para los meses de enero, marzo y abril tienen un comportamiento atípico que puede ser explicado por el arrastre o destrucción de sellos presentes en la superficie del suelo por efecto de la lluvia, la roturación o la pérdida de humedad por insolación. Todos estos factores pueden ser los responsables del incremento en la infiltración y por ende disminución de la escorrentía superficial.

La presencia de niveles altos de escorrentía y suelo erodado en cultivo para los meses de mayo y junio se debe: principalmente a que este sistema tienen un nivel de saturación alto, pues durante esta temporada se presentaron altos volúmenes de precipitación; y a la formación de costras que taponan los macroporos impidiendo la infiltración del agua; favoreciendo la acción erosiva de la lluvia y la escorrentía.

El minisimulador se adaptó a las condiciones presentes en la zona de estudio para evaluar la susceptibilidad a la erosión, permitiendo comparar los tratamientos en suelo desnudo y pastura ante las variables de medición como pérdida de suelo, escorrentía e infiltración.

En las áreas con pastura se puede demostrar el papel fundamental de las coberturas vegetales al mantener un nivel permanente de humedad y el efecto mecánico de las raíces poco profundas que se dispersan con facilidad permitiendo agregar las partículas del suelo y mantener una buena infiltración del agua

El suelo desnudo utilizado en el monocultivo de papa es una de las actividades más utilizadas por los agricultores de la zona, presentando condiciones más desfavorables respecto a: suelo erodado, escorrentía, infiltración; propiedades físicas, químicas, lo que sugiere que este tipo de manejo en suelos genera alto riesgo de erosión.

Las actividades agrícolas generan fuertes roturaciones sometido $\dot{c}$ ? a cambios de humedad, condiciones de alta fluidez y formación de capas compactadas que dañan su estructura, haciéndolo susceptible a la erosión, especialmente cuando hay concentración de lluvias intensas.

La matriz de FEARO muestra que los componentes más afectados por las actividades agropecuarias son: suelo, flora y fauna de una forma adversa ya que las coberturas vegetales de estos ecosistemas son eliminadas o transformadas a pastizales, causando deterioro de las propiedades físicas, químicas y biológicas, que generan degradación de ecosistemas.

\section{BIBLIOGRAFÍA}

Bravo RI, Giraldo RE. Manual de prácticas de química agrícola: Análisis de suelos. Universidad del Cauca. 2 ed. Popayán (Cauca). Facultad de Ciencias Naturales Exactas y de la Educación. Departamento de Química. 2003.

Campo QJ. Evaluación de la susceptibilidad a la erosión hídrica, del contenido de materia orgánica y de las propiedades físicas, en un oxic dystropept bajo seis historias de uso intensivo, en Pescador Cauca, mediante el uso de un minisimulador de lluvia [tesis de pregrado]. Palmira (Valle del cauca). Universidad Nacional de Colombia. Facultad de Ingeniería y Administración Plan Ingeniería Agrícola. 2004

Cobo QL. Diseño, construcción y evaluación de un mini simulador portátil de lluvia para estudios de susceptibilidad a erosión en laderas. Santiago de Cali (Valle del cauca). Universidad del Valle - Universidad Nacional. Facultad de Ingeniería Agrícola. 1998.

Corporación Autónoma Regional del Cauca (CRC). Esquema de Ordenamiento Territorial (EOT). Municipio de Totoró (Cauca). 2002.

--.. Plan Básico de Ordenamiento Territorial (POT). Municipio de Silvia (Cauca) 2000.

Diaz JR, Perez D, Rodriguez Y, Febles JM. Determinación de índices de erosión de suelos aplicando análisis sig para la localidad de San Andrés en la provincia de Pinar del Río. Chapingo (Mexico): Chapingo, Serie ciencias forestales y del ambiente. 2008. 
Diez HJ. Metodologías para la evaluación de la erosión hídrica en cuencas y vertientes. Madrid (España). Universidad de Valladolid. (s.f).

Francis JL, Henry HJ, Barry MO, Andrew FO. Erosion-productivity-soil amendment relationships for wheat over 16 years. Rev sceince direct. 2009 [en linea]. [citado 16 Marzo 2010]. Disponible en

URL: http://www.sciencedirect.com/science/article/B6TC64TX6VWH2/2/a5e226e4dffd11b9be8d98b1b0a396d1.

Food and Agriculture Organization (FAO). Producción de alimentos e impacto ambiental. Documentos técnicos de referencia [en linea]. 1996 [citado 16 Ene 2010]. Disponible en URL :http://www.fao.org/docrep/003/w2612s/w2612s11.htm.

Hudson N. Conservación del suelo. Barcelona (España): Reverte S.A. 1982.

Instituto de Hidrología, Meteorología y Estudios Ambientales de Colombia (IDEAM). Sistema Nacional Ambiental Adscrito al Ministerio de Ambiente, Vivienda y Desarrollo Territorial de Colombia. 2001.

Morgan RP. Factors influencing erosion. Soil erosion and conservation. Jon Wiley \& Sons. New York. 1986.

Nearing MA, Jetten V, Baffaut C, Cerdan O, Couturier A. Modeling esponse of soil erosion and runoff to changes in precipitation and cover. Rev sceince direct. 2005 [citado 15 Marzo 2010]. Disponible en:

URL:http://www.sciencedirect.com/science/article/B6VCG4G1GFKY1/2/0c8d5b32c415a7b7f6036353108f0252

Suarez FM. Estudio detallado de erosión Rio Hondo, La Tetilla, Cuenca Palacé-Robles. Reporte final. Santiago de Cali (Valle del Cauca). 1982. Informe C.V.C. № 82-6.

Torres PA. Evaluación de la susceptibilidad a la erosión y de las características asociadas (infiltración, y escorrentía) de los suelos de ladera en el departamento del Cauca, mediante un mini simulador de lluvia [tesis de pregrado]. Palmira (valle del Cauca). Universidad del Valle, Universidad Nacional de Colombia. Facultad de ingeniería, Plan de ingeniería agrícola. 2000. 